\title{
Common Fixed Point Theorems of Compatible Maps in Complex Valued $b$-Metric Spaces
}

\author{
G. $\operatorname{Yadav}^{1 *}$, R. K. Sharma ${ }^{2}$, G. L. Prajapati ${ }^{3}$ \\ ${ }^{1}$ Institute of Engineering and Technology, Devi Ahilya University, Khandwa Road, Indore- \\ 452017 (M.P.), India \\ ${ }^{2}$ Department of Mathematics, Govt. (Model, Autonomous) Holkar Science College, Indore - \\ 452001 (M.P.), India \\ ${ }^{3}$ Department of Computer Engineering, Institute of Engineering and Technology, Devi \\ Ahilya University, Khandwa Road, Indore-452017 (M.P.), India
}

Received 5 December 2019, accepted in final revised form 18 March 2020

\begin{abstract}
In a paper Mukheimer established some results regarding common fixed point of two self mappings in a complex valued $b$-metric space satisfying a rational type contractive condition. In this paper we have established a common fixed point theorem by making use of compatibility and weak compatibility of self mappings satisfying generalized rational inequality of four mappings as opposed to two self mappings in a complex valued $b$ - metric space. Some concrete examples have also been presented to verify the effectiveness and applicability of established results. Towards the end, an application to solution of Urysohn's integral equations has also been presented to substantiate the usability of the obtained results. Results of this paper generalize the results of Mukheimer and some other earlier results. Theorems approved in this article will be supportable for researchers to work on rational contractions with more than two pair of self mappings.
\end{abstract}

Keywords: Common fixed point; Compatibility; Weakly compatibility; Complex valued $b$-metric spaces.

(C) 2020 JSR Publications. ISSN: 2070-0237 (Print); 2070-0245 (Online). All rights reserved. doi: http://dx.doi.org/10.3329/jsr.v12i4.44374

J. Sci. Res. 12 (4), 431-446 (2020)

\section{Introduction}

A basic and fundamental result namely Banach contraction principle (BCP) was established by Banach [1]. He proved that a contraction map on a complete metric space always possess a unique fixed point. After this interesting result and its various applications, a huge number of generalization of this result are available in

* Corresponding author: yadavgopa10902@gmail.com 
the literature by using different types of contractive conditions in various abstract spaces. By generalizing the Banach contraction principle, Jungck [2] set out tradition of common fixed point of mappings for two commuting mappings on complete metric space. After the result of Jungck [2] many authors introduced many concepts namely weak commutativity, compatibility, weak compatibility of maps (Sessa [3], Jungck [4,5], Jungck and Rhoades [6] etc.) and established results regarding common fixed point theory. In fact commutativity of maps $\Rightarrow$ weak commutativity of maps $\Rightarrow$ Bcompatibility of maps $\Rightarrow$ weak compatibility of maps, but the converse of these implications is not true. In 2011, Azam et al. [7] by introducing the notion of complex valued metric space, gave sufficient condition for the existence of some common fixed point for a pair of maps satisfying rational inequality.

As a generalization of metric space the structure of $b$-metric space was developed by Bakhtin [8]. An analogous to the structure of $b$-metric space, Rao et $a l$. [9] developed the structure of complex valued $b$-metric space and initiated the study of common fixed point of maps. After that number of researchers has proved several results regarding fixed point in context of complex valued $b$-metric space [10-13].

In a paper, Bairagi et al. [12] by generalizing the results of Azam et al. [7], Bhatt et al. [14], Rouzkard et al. [15] and others, proved some common fixed point theorem in complex valued $b$-metric space for a pair of mappings satisfying contractive conditions involving rational inequalities.

The aim of this paper is to prove some results regarding common fixed point of maps, by using the notion of compatibility and weak compatibility of maps in complex valued $b$-metric space satisfying contractive conditions involving rational expression. Further we applied our results to find the solution of Uryshon's integral equations.

\section{Preliminaries}

We recall some basic definition and results which will be utilized in our subsequent discussion.

Definition 1. [7] Let $\mathbb{C}$ be the set of complex numbers and $z_{1}, z_{2} \in \mathbb{C}$. Define a partial order $\lesssim$ on $\mathbb{C}$ as: $z_{1} \lesssim z_{2}$ if $\operatorname{Re}\left(z_{1}\right) \leq \operatorname{Re}\left(z_{2}\right), \operatorname{Im}\left(z_{1}\right) \leq \operatorname{Im}\left(z_{2}\right)$. It follows that $z_{1} \precsim z_{2}$ if one of the following conditions holds:

(i) $\operatorname{Re}\left(z_{1}\right)=\operatorname{Re}\left(z_{2}\right)$ and $\operatorname{Im}\left(z_{1}\right)=\operatorname{Im}\left(z_{2}\right)$

(ii) $\operatorname{Re}\left(z_{1}\right)<\operatorname{Re}\left(z_{2}\right)$ and $\operatorname{Im}\left(z_{1}\right)=\operatorname{Im}\left(z_{2}\right)$

(iii) $\operatorname{Re}\left(z_{1}\right)=\operatorname{Re}\left(z_{2}\right)$ and $\operatorname{Im}\left(z_{1}\right)<\operatorname{Im}\left(z_{2}\right)$

(iv) $\operatorname{Re}\left(z_{1}\right)<\operatorname{Re}\left(z_{2}\right)$ and $\operatorname{Im}\left(z_{1}\right)<\operatorname{Im}\left(z_{2}\right)$

We write $z_{1} \precsim z_{2}$ if $z_{1} \neq z_{2}$ and one of (ii) and (iii) is satisfied and we write $z_{1} \prec z_{2}$ if only (iv) is satisfied. 
Here we note the following holds trivially:

(i) If $0 \lesssim z_{1} \precsim z_{2}$ then $\left|z_{1}\right|<\left|z_{2}\right|$;

(ii) If $z_{1} \prec z_{2}$ and $z_{2} \prec z_{3}$ then $z_{1} \prec z_{3}$;

(iii) If $a, b \in \mathbb{R}$ and $a \leq b$ then $a z \lesssim b z$ for all $z \in \mathbb{C}$;

(iv) If $a, b \in \mathbb{R}$ and $0 \leq a \leq b$ and $z_{1} \lesssim z_{2}$

implies $a z_{1} \precsim b z_{2}$.

Definition 2. [7] Let $X$ be a nonempty set. A function $d: X \times X \rightarrow \mathbb{C}$ is called a complex valued metric on $X$ if for all $z_{1}, z_{2}, z_{3} \in X$ the following conditions are satisfied.

(CVM 1) $0 \lesssim d\left(z_{1}, z_{2}\right)$ and $d\left(z_{1}, z_{2}\right)=0$ if and only if $z_{1}=z_{2}$;

$(\mathrm{CVM} 2) \quad d\left(z_{1}, z_{2}\right)=d\left(z_{2}, z_{1}\right)$;

(CVM3) $d\left(z_{1}, z_{2}\right) \lesssim d\left(z_{1}, z_{3}\right)+d\left(z_{3}, z_{2}\right)$.

Then the pair $(X, d)$ is called a complex valued metric space.

Example 3. [16] Let $X=\mathbb{C}$. Define the mapping $d: X \times X \rightarrow \mathbb{C}$ by

$$
d\left(z_{1}, z_{2}\right)=i\left|z_{1}-z_{2}\right|, \forall z_{1}, z_{2} \in X \text {. }
$$

Then $(X, d)$ is a complex valued metric space.

Example 4. [17] Let $X=\mathbb{C}$. Define the mapping $d: X \times X \rightarrow \mathbb{C}$ by

$$
d\left(z_{1}, z_{2}\right)=e^{i k}\left|z_{1}-z_{2}\right| \text {, where } k \in \mathbb{R}, \forall z_{1}, z_{2} \in X \text {. }
$$

Then $(X, d)$ is a complex valued metric space.

Definition 5. [9] Let $X$ be a nonempty set and $s \geq 1$ be a given real number. A function $d: X \times X \rightarrow \mathbb{C}$ is called a complex valued $b$-metric on $X$ if for all $z_{1}, z_{2}, z_{3} \in X$ the following conditions are satisfied:

(CVbM 1) $\quad 0 \lesssim d\left(z_{1}, z_{2}\right)$ and $d\left(z_{1}, z_{2}\right)=0$ if and only if $z_{1}=z_{2}$;

(CVbM 2) $\quad d\left(z_{1}, z_{2}\right)=d\left(z_{2}, z_{1}\right)$;

(CVbM 3) $\quad d\left(z_{1}, z_{2}\right) \precsim s\left[d\left(z_{1}, z_{3}\right)+d\left(z_{3}, z_{2}\right)\right]$.

Then the pair $(X, d)$ is called a complex valued $b$-metric space.

Example 6. [9] Let $X=[0,1]$. Define the mapping $d: X \times X \rightarrow \mathbb{C}$ by

$$
d(x, y)=|x-y|^{2}+i|x-y|^{2}
$$

Then $(X, d)$ is a complex valued $b$-metric space with $s=2$.

Example 7. [10] Let $X=\mathbb{C}$. Define a function $d: X \times X \rightarrow \mathbb{C}$ such that $d\left(z_{1}, z_{2}\right)=\left|x_{1}-x_{2}\right|^{2}+i\left|y_{1}-y_{2}\right|^{2}, \forall z_{1}, z_{2} \in X$, where $z_{1}=x_{1}+i y_{1}$, $z_{2}=x_{2}+i y_{2}$.

Then $(X, d)$ is a complex valued $b$-metric space with $s=2$. 
Example 8. Let $(X, d)$ be a complex valued metric space and $\rho\left(z_{1}, z_{2}\right)=$ $\left(d\left(z_{1}, z_{2}\right)\right)^{p}$, where $p \geq 1$ is a real number. Then $(X, \rho)$ is a complex valued $b$ metric space.

(i) Since $d\left(z_{1}, z_{2}\right) \gtrsim 0 \Rightarrow\left(d\left(z_{1}, z_{2}\right)\right)^{p} \gtrsim 0 \Rightarrow \rho\left(z_{1}, z_{2}\right) \gtrsim 0$

Moreover, $\rho\left(z_{1}, z_{2}\right)=0 \Leftrightarrow\left(d\left(z_{1}, z_{2}\right)\right)^{p}=0$

$$
\begin{aligned}
& \Leftrightarrow d\left(z_{1}, z_{2}\right)=0 \\
& \Leftrightarrow z_{1}=z_{2} \quad \forall z_{1}, z_{2} \in X .
\end{aligned}
$$

(ii) $\rho\left(z_{1}, z_{2}\right)=\left(d\left(z_{1}, z_{2}\right)\right)^{p}=\left(d\left(z_{2}, z_{1}\right)\right)^{p}=\rho\left(z_{2}, z_{1}\right)$.

(iii) if $a \gtrsim 0, b \succsim 0$ are complex numbers and $p \geq 1$ then $(a+b)^{p} \lesssim 2^{p}\left(a^{p}+b^{p}\right)$.

Case I: $a \gtrsim b$ then $a+b \precsim a+a=2 a$

$\Rightarrow(a+b)^{p} \lesssim 2^{p} a^{p} \lesssim 2^{p}\left(a^{p}+b^{p}\right)$.

Case II: $b \gtrsim a$ then $a+b \lesssim b+b=2 b$.

$\Rightarrow(a+b)^{p} \lesssim 2^{p} b^{p} \lesssim 2^{p}\left(a^{p}+b^{p}\right)$.

Therefore in both cases we have $(a+b)^{p} \lesssim 2^{p}\left(a^{p}+b^{p}\right)$.

Then $\rho\left(z_{1}, z_{2}\right)=\left(d\left(z_{1}, z_{2}\right)\right)^{p} \lesssim\left(d\left(z_{1}, z_{3}\right)+d\left(z_{3}, z_{2}\right)\right)^{p}$

$$
\begin{aligned}
& \lesssim 2^{p}\left(\left(d\left(z_{1}, z_{3}\right)\right)^{p}+\left(d\left(z_{3}, z_{2}\right)\right)^{p}\right) \\
& =2^{p}\left[\rho\left(z_{1}, z_{3}\right)+\rho\left(z_{3}, z_{2}\right)\right] .
\end{aligned}
$$

Hence $(X, \rho)$ is a complex valued $b$-metric space with $s=2^{p}$.

Definition 9. [9] Let $(X, d)$ be a complex valued $b$-metric space. Consider the following:

(i) A point $x \in X$ is called interior point of a set $A \subseteq X$ whenever there exists $0<r \in \mathbb{C}$ such that

$B(x, r)=\{y \in X: d(x, y) \prec r\} \subseteq A$.

(ii) A point $x \in X$ is called a limit point of a set $A \subseteq X$ whenever, for every $0 \prec r \in \mathbb{C}, B(x, r) \cap(A-X) \neq \varnothing$.

(iii) A subset $B \subseteq X$ is called open whenever each element of $B$ is an interior point of $B$.

(iv) A subset $B \subseteq X$ is called closed whenever each limit point of $B$ belongs to $B$.

(v) The family $F=\{B(x, r): x \in X$, and $0 \prec r\}$ is a sub basis for a topology on $X$.

This topology is denoted by $\tau_{c}$. Indeed, the topology $\tau_{c}$ is Hausdorff.

Definition 10. [9] Let $(X, d)$ be a complex valued $b$-metric space and $\left\{z_{n}\right\}$, a sequence in $X$ and

$z \in X$. Consider the following:

(i) If for every $c \in \mathbb{C}$ with $0 \prec c$ there is $\mathrm{N} \in \mathbb{N}$ such that, for all $n \geq N, d\left(z_{n}, z\right) \prec$ $c$, then $\left\{z_{n}\right\}$ is said to be convergent, $\left\{z_{n}\right\}$ converges to $z$, and $z$ is the limit point of $\left\{z_{n}\right\}$. We denote this $\lim _{n \rightarrow \infty} z_{n}=z$ and $\left\{z_{n}\right\} \rightarrow z$ as $n \rightarrow \infty$.

(ii) If for every $c \in \mathbb{C}$ with $0 \prec c$ there is $\mathrm{N} \in \mathbb{N}$ such that, for all $n>N, d\left(z_{n}, z_{n+m}\right) \prec c$, where $\mathrm{m} \in \mathbb{N}$, then $\left\{z_{n}\right\}$ is said to be a Cauchy sequence.

(iii) If every Cauchy sequence is convergent in $(X, d)$, then $(X, d)$ is said to be a complete complex valued $b$-metric space. 
Lemma 11. [9] Let $(X, d)$ be a complex valued $b$-metric space and let $\left\{z_{n}\right\}$ be a sequence in $X$. Then $\left\{z_{n}\right\}$ converges to $z$ if and only if $\left|d\left(z_{n}, z\right)\right| \rightarrow 0$, as $n \rightarrow \infty$.

Lemma 12. [9] Let $(X, d)$ be a complex valued $b$-metric space and let $\left\{z_{n}\right\}$ be a sequence in $X$. Then $\left\{z_{n}\right\}$ is a Cauchy Sequence if and only if $\left|d\left(z_{n}, z_{n+m}\right)\right| \rightarrow 0$ as $n \rightarrow \infty$, where $m \in N$.

Definition 13. Two self maps $S$ and $T$ of a complex valued $b$-metric space $(X, d)$ are weakly commuting if $|d(S T z, T S z)| \leq|d(S z, T z)|, \forall z \in X$.

Definition 14. Two self-maps $S$ and $T$ of a complex valued $b$-metric space $(X, d)$ are compatible if

$\lim _{n \rightarrow \infty}\left|d\left(S T z_{n}, T S z_{n}\right)\right| \rightarrow 0$ whenever $\left\{z_{n}\right\}$ is a sequence in $X$ such that $\lim _{n \rightarrow \infty} S z_{n}=\lim _{n \rightarrow \infty} T z_{n}=z$ for some $z \in X$.

Definition 15. [9] Two self maps $S$ and $T$ of a complex valued $b$-metric space $(X, d)$ are weakly compatible if $S z=T z$ implies that $T S z=S T z$.

Definition 16. A function $T$ defined on a complex valued $b$-metric space $(X, d)$ is called continuous at a point $z_{0} \in X$ if for every $\varepsilon>0$ there exist $\delta>0$ such that $\left|d\left(T z, T z_{0}\right)\right|<\varepsilon$ for all $z \in X$ with $\left|d\left(z, z_{0}\right)\right|<\delta$. i.e. $\lim _{z \rightarrow z_{0}}\left|d\left(T z, T z_{0}\right)\right|=0$.

Proposition 17. Let $S$ and $T$ be two self mappings defined on a complex valued $b$ metric space $(X, d)$. Then the commutativity of $S$ and $T$ implies weak commutativity but the converse is not always true.

Proof. If $\mathrm{S}$ and $\mathrm{T}$ are two self maps on a complex valued $b$-metric space. If $S$ and $T$ are commuting maps then $S T x=T S x \forall x \in X$, therefore $|d(S T x, T S x)|=0$. Then we have

$0=|d(S T x, T S x)| \leq|d(S x, T x)|$ is true. i.e., $S$ and $T$ are weakly commuting maps. For converse we have the following example:

Let $(X, d)$ be a complex valued $b$-metric space, where $X=[0,1]$ and $d: X \times X \rightarrow$ $\mathbb{C}$

defined by $d(x, y)=|x-y|^{2}+i|x-y|^{2}, \forall x, y \in X$.

Define self maps $S$ and $T$ on $X$ by $T x=\frac{x}{x+1}$ and $S x=\frac{x}{x+2} \forall x \in X$.

Then we see that $T(S x)=T\left(\frac{x}{x+2}\right)=\frac{x}{2 x+2}$ and $S(T x)=S\left(\frac{x}{x+1}\right)=\frac{x}{3 x+2}$.

Therefore, $S T \neq T S$ i.e. the mappings $S$ and $T$ are not commuting. Now

$$
d(S T x, T S x)=\left|\frac{x}{3 x+2}-\frac{x}{2 x+2}\right|^{2}+i\left|\frac{x}{3 x+2}-\frac{x}{2 x+2}\right|^{2}=\left|\frac{x}{3 x+2}-\frac{x}{2 x+2}\right|^{2}(1+i)
$$


$\Rightarrow|d(S T x, T S x)|=\left.\frac{1}{4}|| \frac{-x^{2}}{(3 x+2)(x+1)}\right|^{2}(1+i) \mid$.

Also, $d(S x, T x)=\left|\frac{x}{(x+2)}-\frac{x}{(x+1)}\right|^{2}+i\left|\frac{x}{(x+2)}-\frac{x}{(x+1)}\right|^{2}$

$\Rightarrow|d(S x, T x)|=\left|\frac{-x}{(x+2)(x+1)}\right|^{2}|1+i|$.

Hence, $|d(S T x, T S x)| \leq|d(S x, T x)| \forall x \in X$. i.e. $S$ and $T$ are weakly commuting maps. Therefore weakly commutativity does not imply commutativity of maps.

Proposition 18. Let $S$ and $T$ be two self mappings defined on a complex valued $b$ metric space $(X, d)$. Then the weak commutativity of $S$ and $T$ implies compatibility but the converse is not always true.

Proof. If $S$ and $T$ are two self maps of a complex valued $b$-metric space. If $S$ and $T$ are weakly commuting maps then $|d(S T x, T S x)| \leq|d(S x, T x)|, \forall x \in X$.

Now we take a sequence $\left\{x_{n}\right\}$ such that $S x_{n}, T x_{n} \rightarrow t$ as $n \rightarrow \infty$ for some $t \in X$.

Then

$\left|d\left(S T x_{n}, T S x_{n}\right)\right| \leq\left|d\left(S x_{n}, T x_{n}\right)\right| \rightarrow 0$ as $n \rightarrow \infty$. i.e. $S$ and $T$ are compatible maps.

For the converse part, we consider the following example:

Let $(X, d)$ be a complex valued $b$-metric space where $X=[0,1]$ and $d: X \times X \rightarrow$ $\mathbb{C}$ defined by

$d(x, y)=|x-y|^{2}+i|x-y|^{2} \forall x, y \in X$. Define self maps $S$ an $T$ on $X$ by

$T x=x^{3}$ and $S x=2 x^{3} \forall x \in X$. Then we see that

$T(S x)=T\left(2 x^{3}\right)=8 x^{9}$ and $S(T x)=S\left(x^{3}\right)=2 x^{9}$

$d(S T x, T S x)=d\left(2 x^{9}, 8 x^{9}\right)=\left|2 x^{9}-8 x^{9}\right|^{2}+i\left|2 x^{9}-8 x^{9}\right|^{2}=36\left|x^{9}\right|^{2}(1+i)$

$\Rightarrow|d(S T x, T S x)|=\left.36|| x^{9}\right|^{2}|(1+i)| \mid$

$d(S x, T x)=d\left(2 x^{3}, x^{3}\right)=\left|2 x^{3}-x^{3}\right|^{2}+i\left|2 x^{3}-x^{3}\right|^{2}=\left|x^{3}\right|^{2}(1+i)$

$\Rightarrow|d(S x, T x)|=\left|-x^{3}\right|^{2}|(1+i)|$. Therefore we have $|d(S T x, T S x)| \not$ $|d(S x, T x)|$ i.e. $\mathrm{S}$ and $\mathrm{T}$ are not weakly commuting.

But if we take a sequence defined by $x_{n}=\frac{1}{n}$ then $x_{n} \rightarrow 0$ as $n \rightarrow \infty$. Now $S x_{n}=2\left(x_{n}\right)^{3}=2\left(\frac{1}{n}\right)^{3} \rightarrow 0$ as $n \rightarrow \infty$ and $T x_{n}=\left(x_{n}\right)^{3}=\left(\frac{1}{n}\right)^{3} \rightarrow 0$ as $n \rightarrow \infty$ $d\left(S T x_{n}, T S x_{n}\right)=\left|2 x_{n}{ }^{9}-8 x_{n}{ }^{9}\right|^{2}\left|2 x_{n}{ }^{9}-8 x_{n}{ }^{9}\right|^{2}=36\left|x_{n}{ }^{9}\right|^{2}(1+i)+i$ $\Rightarrow\left|d\left(S T x_{n}, T S x_{n}\right)\right|=36\left|x_{n}{ }^{9}\right|^{2}|(1+i)|=36 \sqrt{2}\left|x_{n}{ }^{9}\right|^{2} \rightarrow 0$ as $n \rightarrow \infty$. Hence $S$ and $\mathrm{T}$ are compatible maps.

Proposition 19. Let $S$ and $T$ be two self mappings defined on a complex valued $b$ metric space $(X, d)$. Then the compatibility of $S$ and $T$ implies weak compatibility but the converse is not always true.

Proof. Let $S$ and $T$ be two self maps defined on a complex valued b-metric space $(X, d)$. Suppose that $S$ and $T$ are compatible maps and $S x=T x$ for some $x \in X$. For every $x \in X$, consider the constant sequence $x_{n}=x$ for all $n \in \mathbb{N}$. then $S x_{n}=$ 
$T x_{n} \rightarrow S x$ or $T x$ as $n \rightarrow \infty$ and by the compatibility of $S$ and $T$ we have $|d(S T x, T S x)|=\left|d\left(S T x_{n}, T S x_{n}\right)\right| \rightarrow 0$ as $n \rightarrow \infty$. Hence, STx $=T S x$, i.e. $S$ and $T$ are weakly compatible maps, for the converse part we consider the following example.

Let $(X, d)$ be a complex valued $b$-metric space with the mapping where $X=[0,2]$ and $d: X \times X \rightarrow \mathbb{C}$

defined by $d(x, y)=|x-y|^{2}+i|x-y|^{2}, \forall x, y \in X$.

Define self maps $S$ and $T$ on $X$ by $S x=\left\{\begin{array}{c}1, x=1 \\ 2, \text { otherwise }\end{array}\right.$ and $T x=\left\{\begin{array}{c}1, x=1,2 \\ x, \text { otherwise }\end{array}\right.$

Then 1 is the only coincidence point of $S$ and $T$ i.e. $S(1)=1=T(1)$ and we see that

$S T(1)=S(1)=1, T S(1)=T(1)=1$.

i.e. $S T(1)=T S(1)$ the maps $S$ and $T$ are weakly compatible.

On the other hand, if we take a sequence $\left\{x_{n}\right\}$, defined by $x_{n}=\left(2-\frac{1}{n}\right) \rightarrow 2$ as $n \rightarrow \infty$ and

$S x_{n} \rightarrow 2, T x_{n}=x_{n} \rightarrow 2$ as $n \rightarrow \infty$. But,

$S T x_{n}=S\left(2-\frac{1}{n}\right)=2$ and $T S x_{n}=T(2)=1$ and so $\left|d\left(S T x_{n}, T S x_{n}\right)\right|=|2-1|^{2}+i|2-1|^{2}=1+i \nrightarrow 0$ as $n \rightarrow \infty$.

Hence $S$ and $T$ are not compatible maps.

Also one can not find a sequence $\left\{x_{n}\right\}$ such that $\lim _{n \rightarrow \infty} S x_{n}=\lim _{n \rightarrow \infty} T x_{n}=$ $x \in X$ for some $x \in X$,

such that $\left|d\left(S T x_{n}, T S x_{n}\right)\right| \rightarrow 0$ as $n \rightarrow \infty$. Hence $S$ and $T$ are not compatible maps.

Lemma 20. Let $S$ and $T$ be compatible mappings from a complex valued $b$-metric space $(X, d)$ into itself. Suppose that $\lim _{n \rightarrow \infty} S x_{n}=x$ for some $x \in X$ and if $S$ is continuous. Then $\lim _{n \rightarrow \infty} T S x_{n}=S x$.

Proof. If $\lim _{n \rightarrow \infty} T x_{n}=x, \lim _{n \rightarrow \infty} S T x_{n}=S x$ by continuity of $S$. But if $\lim _{n \rightarrow \infty} S x_{n}=x$.

Then since $d\left(T S x_{n}, S x\right) \lesssim s\left[d\left(T S x_{n}, S T x_{n}\right)+d\left(S T x_{n}, S x\right)\right]$ implies that $\left|d\left(T S x_{n}, S x\right)\right| \leq s\left[\left|d\left(T S x_{n}, S T x_{n}\right)\right|+\left|d\left(S T x_{n}, S x\right)\right|\right]$

Now by the compatibility of $S$ and $T$ we have $\left|d\left(T S x_{n}, S T x_{n}\right)\right| \rightarrow 0$ as $n \rightarrow \infty$, and $\lim _{n \rightarrow \infty} S T x_{n}=S x$.

Then letting $n \rightarrow \infty$ in (1), we have $\left|d\left(T S x_{n}, S x\right)\right| \rightarrow 0$ yields that $\lim _{n \rightarrow \infty} T S x_{n}=$ $S x$.

Mukheimer [13] proved the following results:

Theorem 21. [10] Let $(X, d)$ be a complete complex valued $b$-metric space with the coefficient $s \geq 1$ and $S, T: X \rightarrow X$ be mapping satisfying $d(S z, T w) \lesssim \lambda d(z, w)+\mu \frac{d(z, S z) d(w, T w)}{1+d(z, w)}$

for all $z, w \in X$, where $\lambda, \mu$ are non negative real numbers with $s \lambda+\mu<1$. then $S, T$ have a unique common fixed point in $X$. 
Theorem 22. [10] Let $(X, d)$ be a complete complex valued $b$-metric space with the coefficient

$s \geq 1$ and let $S, T: X \rightarrow X$ be mappings satisfying:

$d(S z, T w) \lesssim \alpha \frac{d(z, S z) d(z, T w)+d(w, S z) d(w, T w)}{d(z, T w)+d(w, S z)}$

for all $z, w \in X$, where $\alpha$, a non negative real number with $s \alpha \in[0,1)$. Then $S, T$ have a unique common fixed point in $X$.

\section{Main Results}

Here by using the notion of compatibility and weak compatibility maps, we generalize the above results by taking four maps as opposed to two maps.

Theorem 23. Let $(X, d)$ be a complete complex valued $b$-metric space and mappings $A, B, S$ and $T$ satisfying.

(i) $S(X) \subseteq B(X), T(X) \subseteq A(X)$

(ii) $\quad d(S z, T w) \precsim$

$\alpha d(A z, B w)+\beta \frac{d(A z, S z) d(B w, T w)}{1+d(A z, B w)}+\gamma \frac{d(A z, S z) d(A z, T w)+d(B w, S z) d(B w, T w)}{d(A z, T w)+d(B w, S z)}$

where $d(A z, T w)+d(B w, S z) \neq 0$.

$d(S z, T w)=0$ if $d(A z, T w)+d(B w, S z)=0$.

for all $z, w$ in $X$ where $\alpha, \beta, \gamma$ are non negative reals with $s \alpha+\beta+s \gamma<1$.

(iii) Suppose that $A$ is continuous, pair $(S, A)$ is compatible and $(T, B)$ is weak compatible.

OR

(iv) $T$ is continuous, pair $(S, A)$ is weak compatible and $(T, B)$ is compatible.

Then $A, B, S$ and $T$ have unique common fixed point in $X$.

Proof. Suppose $z_{0}$ be an arbitrary point in $X$ we define a sequence $\left\{w_{2 n}\right\}$ in $X$ such that

$w_{2 n}=S z_{2 n}=B z_{2 n+1} \quad ; \quad w_{2 n+1}=T z_{2 n+1}=A z_{2 n+2}$, for $n=0,1,2, \ldots$

Now from (ii), we have

$$
\begin{aligned}
& d\left(w_{2 n}, w_{2 n+1}\right)=d\left(S z_{2 n}, T z_{2 n+1}\right) \\
& \lesssim \alpha d\left(A z_{2 n}, B z_{2 n+1}\right)+\beta \frac{d\left(A z_{2 n}, S z_{2 n}\right) d\left(B z_{2 n+1}, T z_{2 n+1}\right)}{1+d\left(A z_{2 n}, B z_{2 n+1}\right)} \\
& +\gamma \frac{d\left(A z_{2 n}, S z_{2 n}\right) d\left(A z_{2 n}, T z_{2 n+1}\right)+d\left(B z_{2 n+1}, S z_{2 n}\right) d\left(B z_{2 n+1}, T z_{2 n+1}\right)}{d\left(A z_{2 n}, T z_{2 n+1}\right)+d\left(B z_{2 n+1}, S z_{2 n}\right)}
\end{aligned}
$$

Since $d\left(A z_{2 n}, T z_{2 n+1}\right)+d\left(B z_{2 n+1}, S z_{2 n}\right)=d\left(w_{2 n-1}, w_{2 n+1}\right)+d\left(w_{2 n}, w_{2 n}\right) \neq 0$. which implies that

$$
\begin{gathered}
\left|d\left(w_{2 n}, w_{2 n+1}\right)\right| \leq \alpha\left|d\left(w_{2 n-1}, w_{2 n}\right)\right|+\beta \frac{\left|d\left(w_{2 n-1}, w_{2 n}\right)\right|\left|d\left(w_{2 n}, w_{2 n+1}\right)\right|}{\left|1+d\left(w_{2 n-1}, w_{2 n}\right)\right|} \\
+\gamma \frac{\left|d\left(w_{2 n-1}, w_{2 n}\right)\right|\left|d\left(w_{2 n-1}, w_{2 n+1}\right)\right|+\left|d\left(w_{2 n}, w_{2 n}\right)\right|\left|d\left(w_{2 n}, w_{2 n+1}\right)\right|}{\left|d\left(w_{2 n-1}, w_{2 n+1}\right)+d\left(w_{2 n}, w_{2 n}\right)\right|} \\
\leq \alpha\left|d\left(w_{2 n-1}, w_{2 n}\right)\right|+\beta\left|d\left(w_{2 n}, w_{2 n+1}\right)\right|+\gamma\left|d\left(w_{2 n-1}, w_{2 n}\right)\right| \\
\left|d\left(w_{2 n}, w_{2 n+1}\right)\right| \leq\left(\frac{\alpha+\gamma}{1-\beta}\right)\left|d\left(w_{2 n-1}, w_{2 n}\right)\right| .
\end{gathered}
$$


Similarly

Since

$$
\begin{gathered}
d\left(w_{2 n+1}, W_{2 n+2}\right) \lesssim \alpha d\left(A z_{2 n+1}, B z_{2 n+2}\right)+\beta \frac{d\left(A z_{2 n+1}, S z_{2 n+1}\right) d\left(B z_{2 n+2}, T z_{2 n+2}\right)}{1+d\left(A z_{2 n+1}, B z_{2 n+2}\right)} \\
+\gamma \frac{d\left(A z_{2 n+1}, S z_{2 n+1}\right) d\left(A z_{2 n+1}, T z_{2 n+2}\right)+d\left(B z_{2 n+2}, S z_{2 n+1}\right) d\left(B z_{2 n+2}, T z_{2 n+2}\right)}{d\left(A z_{2 n+1}, T z_{2 n+2}\right)+d\left(B z_{2 n+2}, S z_{2 n+1}\right)}
\end{gathered}
$$

$d\left(A z_{2 n+1}, T z_{2 n+2}\right)+d\left(B z_{2 n+2}, S z_{2 n+1}\right)=d\left(w_{2 n}, w_{2 n+2}\right)+d\left(w_{2 n+1}, w_{2 n+1}\right) \neq$ 0 .

$$
\begin{gathered}
\left|d\left(w_{2 n+1}, w_{2 n+2}\right)\right| \leq \alpha\left|d\left(w_{2 n}, w_{2 n+1}\right)\right|+\beta \frac{\left|d\left(w_{2 n}, w_{2 n+1}\right)\right|\left|d\left(w_{2 n+1}, w_{2 n+2}\right)\right|}{\left|1+d\left(w_{2 n}, w_{2 n+1}\right)\right|} \\
+\gamma \frac{\left|d\left(w_{2 n}, w_{2 n+1}\right)\right|\left|d\left(w_{2 n}, w_{2 n+2}\right)\right|+\left|d\left(w_{2 n+1}, w_{2 n+1}\right)\right|\left|d\left(w_{2 n+1}, w_{2 n+2}\right)\right|}{\left|d\left(w_{2 n}, w_{2 n+2}\right)+d\left(w_{2 n+1}, w_{2 n+1}\right)\right|} \\
\leq \alpha\left|d\left(w_{2 n}, w_{2 n+1}\right)\right|+\beta\left|d\left(w_{2 n+1}, w_{2 n+2}\right)\right|+\gamma\left|d\left(w_{2 n}, w_{2 n+1}\right)\right| \\
\leq\left(\frac{\alpha+\gamma}{1-\beta}\right)\left|d\left(w_{2 n}, w_{2 n+1}\right)\right| .
\end{gathered}
$$

Since if $s \alpha+\beta+s \gamma<1$, where $s \geq 1$ then $\frac{s(\alpha+\gamma)}{1-\beta}<1$ or $s \delta<1$ where $\left(\delta=\frac{\alpha+\gamma}{1-\beta}\right)$.

Therefore for all $n \geq 0$, we have $\left|d\left(w_{2 n+1}, w_{2 n+2}\right)\right| \leq \delta\left|d\left(w_{2 n}, w_{2 n+1}\right)\right| \leq \delta^{2}\left|d\left(w_{2 n-1}, w_{2 n}\right)\right| \leq \ldots \leq$ $\delta^{2 n+1}\left|d\left(w_{0}, w_{1}\right)\right|$

Now for any $m>n, \quad m, n \in N$ and by (CVbM 3), we have

$$
\begin{gathered}
\left|d\left(w_{2 n}, w_{2 m}\right)\right| \leq s\left|d\left(w_{2 n}, w_{2 n+1}\right)\right|+s\left|d\left(w_{2 n+1}, w_{2 m}\right)\right| \\
\leq s\left|d\left(w_{2 n}, w_{2 n+1}\right)\right|+s^{2}\left|d\left(w_{2 n+1}, w_{2 n+2}\right)\right|+s^{2}\left|d\left(w_{2 n+2}, w_{2 m}\right)\right| \\
\leq s\left|d\left(w_{2 n}, w_{2 n+1}\right)\right|+s^{2}\left|d\left(w_{2 n+1}, w_{2 n+2}\right)\right|+s^{3}\left|d\left(w_{2 n+2}, w_{2 n+3}\right)\right|+\cdots \\
\ldots \ldots \ldots+s^{2 m-2 n-2}\left|d\left(w_{2 m-3}, w_{2 m-2}\right)\right|+s^{2 m-2 n-1}\left|d\left(w_{2 m-2}, w_{2 m-1}\right)\right|+ \\
s^{2 m-2 n}\left|d\left(w_{2 m-1}, w_{2 m}\right)\right| .
\end{gathered}
$$

By using (3)

$$
\begin{aligned}
\left|d\left(w_{2 n}, w_{2 m}\right)\right| \leq & s \delta^{2 n}\left|d\left(w_{0}, w_{1}\right)\right|+s^{2} \delta^{2 n+1}\left|d\left(w_{0}, w_{1}\right)\right|+s^{3} \delta^{2 n+2}\left|d\left(w_{0}, w_{1}\right)\right| \\
& +\ldots \ldots+s^{2 m-2 n} \delta^{2 m-1}\left|d\left(w_{0}, w_{1}\right)\right|
\end{aligned}
$$$$
\leq \sum_{i=1}^{2 m-2 n} s^{i+2 n-1} \delta^{i+2 n-1}\left|d\left(w_{0}, w_{1}\right)\right|=\sum_{t=2 n}^{2 m-1} s^{t} \delta^{t}\left|d\left(w_{0}, w_{1}\right)\right|
$$$$
\leq \sum_{t=2 n}^{\infty}(s \delta)^{t}\left|d\left(w_{0}, w_{1}\right)\right| \leq \frac{(s \delta)^{2 n}}{(1-s \delta)}\left|d\left(w_{0}, w_{1}\right)\right| \rightarrow 0 \quad \text { as } m, n \rightarrow \infty \text { (since }
$$

$s \delta<1)$

Hence $\left\{w_{2 n}\right\}$ is a Cauchy sequence in $X$. Since $X$ is complete, therefore $\left\{w_{2 n}\right\}$ converges to point $t$ in $X$ and its subsequences $\left\{S z_{2 n}\right\},\left\{T z_{2 n+1}\right\},\left\{A z_{2 n+2}\right\},\left\{B z_{2 n+1}\right\}$ are also converge to $t$.

Case I Suppose that $A$ is continuous. Then $A^{2} z_{2 n}=A A z_{2 n} \rightarrow A t$. Also by the compatibility of $S$ and $A$, from Lemma $20 S A z_{2 n} \rightarrow A t$.

Using (ii), we have

$$
d\left(S A z_{2 n}, T z_{2 n+1}\right) \lesssim \alpha d\left(A^{2} z_{2 n}, B z_{2 n+1}\right)+\beta \frac{d\left(A^{2} z_{2 n}, S A z_{2 n}\right) d\left(B z_{2 n+1}, T z_{2 n+1}\right)}{1+d\left(A^{2} z_{2 n}, B z_{2 n+1}\right)}
$$




$$
\begin{aligned}
& +\gamma \frac{d\left(A^{2} z_{2 n}, S A z_{2 n}\right) d\left(A^{2} z_{2 n}, T z_{2 n+1}\right)+d\left(B z_{2 n+1}, S A z_{2 n}\right) d\left(B z_{2 n+1}, T z_{2 n+1}\right)}{d\left(A^{2} z_{2 n}, T z_{2 n+1}\right)+d\left(B z_{2 n+1}, S A z_{2 n}\right)} \\
& \quad\left|d\left(S A z_{2 n}, T z_{2 n+1}\right)\right| \leq \alpha\left|d\left(A^{2} z_{2 n}, B z_{2 n+1}\right)\right|+\beta \frac{\left|d\left(A^{2} z_{2 n}, S A z_{2 n}\right)\right|\left|d\left(B z_{2 n+1}, T z_{2 n+1}\right)\right|}{\left|1+d\left(A^{2} z_{2 n}, B z_{2 n+1}\right)\right|} \\
& \quad+\gamma \frac{\left|d\left(A^{2} z_{2 n}, S A z_{2 n}\right)\right|\left|d\left(A^{2} z_{2 n}, T z_{2 n+1}\right)\right|+\left|d\left(B z_{2 n+1}, S A z_{2 n}\right)\right|\left|d\left(B z_{2 n+1}, T z_{2 n+1}\right)\right|}{\left|d\left(A^{2} z_{2 n}, T z_{2 n+1}\right)+d\left(B z_{2 n+1}, S A z_{2 n}\right)\right|}
\end{aligned}
$$

Since $d\left(A^{2} z_{2 n}, T z_{2 n+1}\right)+d\left(B z_{2 n+1}, S A z_{2 n}\right)=d(A t, t)+d(A t, t) \neq 0$.

Letting $n \rightarrow \infty$, we get

$$
\begin{gathered}
|d(A t, t)| \leq \alpha|d(A t, t)|+\beta \frac{|d(A t, A t)||d(t, t)|}{|1+d(A t, t)|}+\gamma \frac{|d(A t, A t)||d(A t, t)|+|d(t, A t)||d(t, t)|}{|d(A t, t)+d(t, A t)|} \\
\Rightarrow(1-\alpha)|d(A t, t)| \leq 0 \quad \text { yields } A t=t .
\end{gathered}
$$

Again using (ii), we get

$$
\begin{aligned}
& d\left(S t, T z_{2 n+1}\right) \lesssim \\
& \alpha d\left(A t, B z_{2 n+1}\right)+\beta \frac{d(A t, S t) d\left(B z_{2 n+1}, T z_{2 n+1}\right)}{1+d\left(A t, B z_{2 n+1}\right)}+ \\
& \gamma \frac{d(A t, S t) d\left(A t, T z_{2 n+1}\right)+d\left(B z_{2 n+1}, S t\right) d\left(B z_{2 n+1}, T z_{2 n+1}\right)}{d\left(A t, T z_{2 n+1}\right)+d\left(B z_{2 n+1}, S t\right)}
\end{aligned}
$$

Since $d\left(A t, T z_{2 n+1}\right)+d\left(B z_{2 n+1}, S t\right)=d(t, t)+d(t, S t) \neq 0$.

Letting $n \rightarrow \infty$, we have

$$
\begin{gathered}
|d(S t, t)| \leq \alpha|d(t, t)|+\beta \frac{|d(t, S t)||d(t, t)|}{|1+d(t, t)|}+\gamma \frac{|d(t, S t)||d(t, t)|+|d(t, S t)||d(t, t)|}{|d(t, t)+d(t, S t)|} \\
\Rightarrow|d(S t, t)| \leq 0 \quad \text { yields } \quad S t=t=A t .
\end{gathered}
$$

Now from (i) since $S(X) \subseteq B(X)$, there exists a point $u$ in $X$ such that $t=S t=$ $B u=A t$.

Then from (ii), we have

$$
\begin{aligned}
& |d(t, T u)|=|d(S t, T u)| \leq \alpha|d(A t, B u)|+\beta \frac{|d(A t, S t)||d(B u, T u)|}{|1+d(A t, B u)|}+ \\
& \gamma \frac{|d(A t, S t)||d(A t, T u)|+|d(B u, S t)||d(B u, T u)|}{|d(A t, T u)+d(B u, S t)|} \\
& \Rightarrow|d(t, T u)| \leq 0 \quad \text { yields } \quad t=T u=B u=S t=A t .
\end{aligned}
$$

Now by weak compatibility of $T$ and $B$, we have $T B u=B T u \Rightarrow T t=B t$.

Again from (ii), we get

$$
\begin{gathered}
d(S t, T t) \lesssim \alpha d(A t, B t)+\beta \frac{d(A t, S t) d(B t, T t)}{1+d(A t, B t)}+\gamma \frac{d(A t, S t) d(A t, T t)+d(B t, S t) d(B t, T t)}{d(A t, T t)+d(B t, S t)} \\
|d(t, B t)|=|d(S t, T t)| \leq \alpha|d(A t, B t)|+\beta \frac{|d(A t, S t)||d(B t, T t)|}{|1+d(A t, B t)|}+ \\
\gamma \frac{|d(A t, S t)||d(A t, T t)|+|d(B t, S t)||d(B t, T t)|}{|d(A t, T t)+d(B t, S t)|} \\
\Rightarrow|d(t, B t)| \leq \alpha|d(t, B t)| \text { or }(1-\alpha)|d(t, B t)| \leq 0 \text { yields } B t=t .
\end{gathered}
$$

Hence $A t=B t=S t=T t=t$, i.e. $t$ is the common fixed point of $A, B, S$ and $T$.

Case II For the 'or' part let $T$ is continuous. Then $T^{2} z_{2 n}=T T z_{2 n} \rightarrow T t$. Also by the compatiblility of $T$ and $B$, from Lemma (2.20) $B T z_{2 n} \rightarrow T t$.

Using (3.1.2), we have

$$
\begin{gathered}
d\left(S z_{2 n}, T^{2} z_{2 n}\right) \lesssim \alpha d\left(A z_{2 n}, B T z_{2 n}\right)+\beta \frac{d\left(A z_{2 n}, S z_{2 n}\right) d\left(B T z_{2 n}, T^{2} z_{2 n}\right)}{1+d\left(A z_{2 n}, B T z_{2 n}\right)} \\
+\gamma \frac{d\left(A z_{2 n}, S z_{2 n}\right) d\left(A z_{2 n}, T^{2} z_{2 n}\right)+d\left(B T z_{2 n}, S z_{2 n}\right) d\left(B T z_{2 n}, T^{2} z_{2 n}\right)}{d\left(A z_{2 n}, T^{2} z_{2 n}\right)+d\left(B T z_{2 n}, S z_{2 n}\right)}
\end{gathered}
$$

Since $d\left(A z_{2 n}, T^{2} z_{2 n}\right)+d\left(B T z_{2 n}, S z_{2 n}\right)=d(t, T t)+d(t, T t) \neq 0$. 


$$
\Rightarrow\left|d\left(S z_{2 n}, T^{2} z_{2 n}\right)\right| \leq \alpha\left|d\left(A z_{2 n}, B T z_{2 n}\right)\right|+\beta \frac{\left|d\left(A z_{2 n}, S z_{2 n}\right) \| d\left(B T z_{2 n}, T^{2} z_{2 n}\right)\right|}{\left|1+d\left(A z_{2 n}, B T z_{2 n}\right)\right|}
$$

$+\gamma \frac{\left|d\left(A z_{2 n}, S z_{2 n}\right)\right|\left|d\left(A z_{2 n}, T^{2} z_{2 n}\right)\right|+\left|d\left(B T z_{2 n}, S z_{2 n}\right)\right|\left|d\left(B T z_{2 n}, T^{2} z_{2 n}\right)\right|}{\left|d\left(A z_{2 n}, T^{2} z_{2 n}\right)+d\left(B T z_{2 n}, S z_{2 n}\right)\right|}$

Letting $n \rightarrow \infty$, we have

$|d(t, T t)| \leq \alpha|d(t, T t)|+\beta \frac{|d(t, t)||d(T t, T t)|}{|1+d(t, T t)|}+\gamma \frac{|d(t, t)||d(t, T t)|+|d(T t, t)||d(T t, T t)|}{|d(t, T t)+d(T t, t)|}$

$\Rightarrow(1-\alpha)|d(t, T t)| \leq 0$ yields $T t=t$.

Now from (i) since $T(X) \subseteq A(X)$, there exist a point $v$ in $X$ such that $t=T t=A v$.

Then from (ii), we have

$d\left(S v, T^{2} z_{2 n}\right) \lesssim$

$\alpha d\left(A v, B T z_{2 n}\right)+\beta \frac{d(A v, S v) d\left(B T z_{2 n}, T^{2} z_{2 n}\right)}{1+d\left(A v, B T z_{2 n}\right)}+$

$\gamma \frac{d(A v, S v) d\left(A v, T^{2} z_{2 n}\right)+d\left(B T z_{2 n}, S v\right) d\left(B T z_{2 n}, T^{2} z_{2 n}\right)}{d\left(A v, T^{2} z_{2 n}\right)+d\left(B T z_{2 n}, S v\right)}$

Since $d\left(A v, T^{2} z_{2 n}\right)+d\left(B T z_{2 n}, S v\right)=d(t, T t)+d(T t, S v) \neq 0$.

$\Rightarrow\left|d\left(S v, T^{2} z_{2 n}\right)\right| \leq \alpha\left|d\left(A z_{2 n}, B T z_{2 n}\right)\right|+\beta \frac{|d(A v, S v)|\left|d\left(B T z_{2 n}, T^{2} z_{2 n}\right)\right|}{\left|1+d\left(A v, B T z_{2 n}\right)\right|}+$

$\gamma \frac{|d(A v, S v)|\left|d\left(A v, T^{2} z_{2 n}\right)\right|+\left|d\left(B T z_{2 n}, S v\right)\right|\left|d\left(B T z_{2 n}, T^{2} z_{2 n}\right)\right|}{\left|d\left(A v, T^{2} z_{2 n}\right)+d\left(B T z_{2 n}, S v\right)\right|}$

Letting $n \rightarrow \infty$, we have

$$
|d(S v, T t)| \leq
$$

$\alpha|d(t, T t)|+\beta \frac{|d(t, S v)||d(T t, T t)|}{|1+d(t, T t)|}+\gamma \frac{|d(t, S v)||d(t, T t)|+|d(T t, S v)||d(T t, T t)|}{|d(t, T t)|+|d(T t, S v)|}$

$|d(S v, t)| \leq \alpha|d(t, t)|+\beta \frac{|d(t, S v)||d(t, t)|}{|1+d(t, t)|}+\gamma \frac{|d(t, S v)||d(t, t)|+|d(t, S v)||d(t, t)|}{|d(t, t)|+|d(t, S v)|}$

$$
\Rightarrow|d(S v, t)| \leq 0, \text { yields } S v=t=T t .
$$

Since $S$ and $A$ are weakly compatible on $X$ and $S v=A v$ and $S A v=A S v \Rightarrow S t=$ $A t$. Using (ii), we have

$$
\begin{aligned}
& d\left(S t, T z_{2 n+1}\right) \lesssim \alpha d\left(A t, B z_{2 n+1}\right)+\beta \frac{d(A t, S t) d\left(B z_{2 n+1}, T z_{2 n+1}\right)}{1+d\left(A t, B z_{2 n+1}\right)}+ \\
& \gamma \frac{d(A t, S t) d\left(A t, T z_{2 n+1}\right)+d\left(B z_{2 n+1}, S t\right) d\left(B z_{2 n+1}, T z_{2 n+1}\right)}{d\left(A t, T z_{2 n+1}\right)+d\left(B z_{2 n+1}, S t\right)}
\end{aligned}
$$

Since $d\left(A t, T z_{2 n+1}\right)+d\left(B z_{2 n+1}, S t\right)=d(A t, t)+d(t, S t) \neq 0$.

$$
\Rightarrow\left|d\left(S t, T z_{2 n+1}\right)\right| \leq \alpha\left|d\left(A t, B z_{2 n+1}\right)\right|+\beta \frac{|d(A t, S t)|\left|d\left(B z_{2 n+1}, T z_{2 n+1}\right)\right|}{\left|1+d\left(A t, B z_{2 n+1}\right)\right|}
$$

$+\gamma \frac{|d(A t, S t)|\left|d\left(A t, T z_{2 n+1}\right)\right|+\left|d\left(B z_{2 n+1}, S t\right)\right|\left|d\left(B z_{2 n+1}, T z_{2 n+1}\right)\right|}{\left|d\left(A t, T z_{2 n+1}\right)+d\left(B z_{2 n+1}, S t\right)\right|}$

Letting $\rightarrow \infty$, we have

$|d(S t, t)| \leq \alpha|d(S t, t)|+\beta \frac{|d(A t, A t)||d(t, t)|}{|1+d(A t, t)|}+\gamma \frac{|d(A t, A t)||d(A t, t)|+|d(t, S t)||d(t, t)|}{|d(A t, t)|+|d(t, S t)|}$

$\Rightarrow(1-\alpha)|d(S t, t)| \leq 0$, yields $S t=t=A t=T t=S v$.

Since $S(X) \subseteq B(X)$, there exists a point $w$ in $X$ such that $t=S t=B w$.

Now, from (ii), we have

$d(t, T w)=d(S t, T w) \lesssim$

$\alpha d(A t, B w)+\beta \frac{d(A t, S t) d(B w, T w)}{1+d(A t, B t)}+\gamma \frac{d(A t, S t) d(A t, T w)+d(B w, S t) d(B w, T w)}{d(A t, T w)+d(B w, S t)}$

$|d(t, T w)| \leq \alpha|d(t, t)|+\beta \frac{|d(A t, t)||d(t, T w)|}{|1+d(A t, t)|}+\gamma \frac{|d(A t, t)||d(A t, T w)|+|d(t, t)||d(t, T w)|}{|d(A t, T w)|+|d(t, S t)|}$ 


$$
|d(t, T w)| \leq \alpha|d(t, t)|+\beta \frac{|d(t, t)||d(t, T w)|}{|1+d(t, t)|}+\gamma \frac{|d(t, t)||d(t, T w)|+|d(t, t)||d(t, T w)|}{|d(t, T w)|+|d(t, S t)|}
$$

$\Rightarrow|d(t, T w)| \leq 0$, yields $t=T w$. Hence $t=S t=B w=T w=T t=A t$.

Since $T$ and $B$ are compatible on $X$ and $T w=B w=t$ then by proposition (18),

$d(B T w, T B w)=0$.

This implies $B t=B T w=T B w=T t$. Hence $S t=T t=A t=B t=t$.

Therefore, $t$ is a common fixed point of $A, B, S$ and $T$.

Now for the uniqueness of $t$, suppose that $t^{*} \neq t$ be another common fixed point of $A, B, S$ and $T$.

Then, from (ii), we have

$$
\begin{gathered}
d\left(t, t^{*}\right)=d\left(S t, T t^{*}\right) \lesssim \\
\alpha d\left(A t, B t^{*}\right)+\beta \frac{d(A t, S t) d\left(B t^{*}, T t^{*}\right)}{1+d(A t, B w)}+\gamma \frac{d(A t, S t) d\left(A t, T t^{*}\right)+d\left(B t^{*}, S t\right) d\left(B t^{*}, T t^{*}\right)}{d\left(A t, T t^{*}\right)+d\left(B t^{*}, S t\right)} \\
\left|d\left(t, t^{*}\right)\right| \leq \alpha\left|d\left(t, t^{*}\right)\right|+\beta \frac{|d(t, t)|\left|d\left(t^{*}, t^{*}\right)\right|}{\left|1+d\left(t, t^{*}\right)\right|}+\gamma \frac{|d(t, t)|\left|d\left(t, t^{*}\right)\right|+\left|d\left(t^{*}, t\right)\right|\left|d\left(t^{*}, t^{*}\right)\right|}{\left|d\left(t, t^{*}\right)\right|+\left|d\left(t^{*}, t\right)\right|} \\
\Rightarrow(1-\alpha)\left|d\left(t, t^{*}\right)\right| \leq 0 \quad \text { which is contradiction. Hence } t=t^{*} .
\end{gathered}
$$

i.e. $t$ is the unique common fixed point of $A, B, S$ and $T$.

On setting $A=B=I$ and $\gamma=0$ in the inequality (ii), we have the following results (Theorem 20 of [10]) as a corollary.

Corollary 24. Let $(X, d)$ be a complete complex valued $b$-metric space with the coefficient $s \geq 1$ and $S, T: X \rightarrow X$ be mapping satisfying

$d(S z, T w) \lesssim \alpha d(z, w)+\beta \frac{d(z, S z) d(w, T w)}{1+d(z, w)}$

for all $z, w \in X$, where $\alpha, \beta$ are non negative real numbers with $s \alpha+\beta<1$. then $S, T$ have a unique common fixed point in $X$.

On setting $A=B=I$ and $\alpha=\beta=0$ in the ineuality (ii), we have the following results (Theorem 21 of [10]) as a corollary.

Corollary 25. Let $(X, d)$ be a complete complex valued $b$-metric space with the coefficient $s \geq 1$ and let $S, T: X \rightarrow X$ be mappings satisfying:

$$
d(S z, T w) \lesssim \gamma \frac{d(z, S z) d(z, T w)+d(z, S w) d(w, T w)}{d(z, T w)+d(w, S z)}
$$

for all $z, w \in X$, where $\gamma$ non negative real number with $s \gamma \in[0,1)$. Then $S, T$ have a unique common fixed point in $X$.

On setting $A=B=I$ and $\gamma=0$ in the inequality (ii), we have the following results (Theorem 4 of [7]) as a corollary.

Corollary 26. Let $(X, d)$ be a complete complex valued $b$-metric space with the coefficient $s=1$ and $S, T: X \rightarrow X$ be mapping satisfying

$d(S z, T w) \lesssim \alpha d(z, w)+\beta \frac{d(z, S z) d(w, T w)}{1+d(z, w)}$

for all $z, w \in X$,where $\alpha, \beta$ are non negative real numbers with $\alpha+\beta<1$. then $S, T$ have a unique common fixed point in $X$.

Example 27. Let $X=[0,1]$ and $d: X \times X \rightarrow \mathbb{C}$ defined by $d(z, w)=$ $i|z-w|^{2} \forall z, w \in X$.

Then $(X, d)$ be a complete complex valued $b$-metric space with the coefficient $s=2$. Now, we define the self mappings $A, B, S, T: X \rightarrow X$ by 
$S z=\frac{z}{2}, B z=z$ and $T z=\frac{z}{4}, A z=\frac{2 z}{3}$ for all $z, w \in X$.

$S(X)=\left[0, \frac{1}{2}\right] \subseteq[0,1]=B(X), T(X)=\left[0, \frac{1}{4}\right] \subseteq\left[0, \frac{2}{3}\right]=A(X)$.

By the definition of self mappings we get condition (i) of the theorem 23. Now consider

$$
\begin{aligned}
& d(S z, T w)=i|S z-T w|^{2}=i\left|\frac{z}{2}-\frac{w}{4}\right|^{2} \\
& d(A z, B w)=i|A z-B w|^{2}=i\left|\frac{2 z}{3}-w\right|^{2} \\
& d(A z, S z)=i|A z-S z|^{2}=i\left|\frac{2 z}{3}-\frac{z}{2}\right|^{2} \\
& d(B w, T w)=i|B w-T w|^{2}=i\left|w-\frac{w}{4}\right|^{2} \\
& d(A z, T w)=i|A z-T w|^{2}=i\left|\frac{2 z}{3}-\frac{w}{4}\right|^{2} \\
& d(B w, S z)=i|B w-S z|^{2}=i\left|w-\frac{z}{2}\right|^{2}
\end{aligned}
$$

For the verification of inequality (ii), it is sufficient to show that $d(S z, T w) \lesssim \alpha d(A z, B w)$ At $z=0$ and $w=0$ the result is obvious.

At $z=0$ and $w=1, d(S z, T w)=\frac{1}{16}=0.0625$ and $d(A z, B w)=1$.

At $z=1$ and $w=0, d(S z, T w)=\frac{1}{4}=0.25$ and $d(A z, B w)=\frac{4}{9}=0.44$.

At $z=1$ and $w=1, d(S z, T w)=\frac{1}{16}=0.0625$ and $d(A z, B w)=\frac{1}{9}=0.1111$.

Hence the inequality

$$
\begin{aligned}
d(S z, T w) \lesssim \alpha d & (A z, B w)+\beta \frac{d(A z, S z) d(B w, T w)}{1+d(A z, B w)} \\
& +\gamma \frac{d(A z, S z) d(A z, T w)+d(B w, S z) d(B w, T w)}{d(A z, T w)+d(B w, S z)}
\end{aligned}
$$

holds good for all $z, w$ in $X$ where $\alpha=\frac{1}{16}, \beta=\frac{1}{6}$ and $\gamma=\frac{1}{8}$. i.e. $2 \alpha+\beta+2 \gamma<$ 1.

Since the commutativity of pairs $(S, A)$ and $(T, B)$ yields the compatibility of $(S, A)$ and weak compatibility of $(T, B)$.

\section{Urysohn Integral Equations}

In this section, we studied [7,18] and some other papers and we apply our result (Theorem 23) to the existence and uniqueness of a common solution of the system of the Urysohn's integral equations.

Theorem 28. Let $X=C\left([a, b], \mathbb{R}^{n}\right)$ and $d: X \times X \rightarrow \mathbb{C}$ be defined by $d(z, w)=\max _{t \in[a, b]}\|z(t)-w(t)\|_{\infty} \sqrt{1+a^{2}} e^{i \tan ^{-1} a}$.

Consider the Urysohn's integral equations

$$
z(t)=\psi_{i}(t)+\int_{a}^{b} K_{i}(t, s, z(s)) d s
$$

where $i=1,2,3,4, a, b \in \mathbb{R}$ with $a \leq b, t \in[a, b], z, \psi_{i} \in C\left([a, b], \mathbb{R}^{n}\right)$.

Suppose $K_{i}:[a, b] \times[a, b] \times \mathbb{R}^{n} \rightarrow \mathbb{R}^{n}$ is a given mapping for each $i=1,2,3,4$. 
Throughout this section, for each $i=1,2,3,4$ and $K_{i}$ in equation (4), we make use the following symbols $\delta_{i}(z(t))=\int_{a}^{b} K_{i}(t, s, z(s)) d s$.

Assume the following conditions hold for each $t \in[a, b]$ :

(a) $\alpha+\beta+\gamma<1$, when $s=1$.

(b) $\delta_{1} z(t)+\psi_{1}(t)+\psi_{4}(t)-\delta_{4}\left[\delta_{1} z(t)+\psi_{1}(t)+\psi_{4}(t)\right]=0$ and

$$
\delta_{2} z(t)+\psi_{2}(t)+\psi_{3}(t)-\delta_{3}\left[\delta_{2} z(t)+\psi_{2}(t)+\psi_{3}(t)\right]=0
$$

(c) $\delta_{1}\left(\delta_{3} z(t)+\psi_{3}(t)\right)+\psi_{1}(t)-\left[\delta_{3}\left(\delta_{1} z(t)+\psi_{1}(t)\right)+\psi_{3}(t)\right]=0$ and $\delta_{2}\left(\delta_{4} z(t)+\psi_{4}(t)\right)+\psi_{2}(t)-\left[\delta_{4}\left(\delta_{2} z(t)+\psi_{2}(t)\right)+\psi_{4}(t)\right]=0$.

(d) $\psi_{1}(t)+3 \psi_{3}(t)+\delta_{1}\left(2 z(t)-\psi_{1}(t)\right)+2 \delta_{3} z(t)+\delta_{3}\left(2 z(t)-\delta_{3} z(t)-\right.$ $\left.\psi_{3}(t)\right)=4 z(t)$ and

$$
\begin{aligned}
\psi_{2}(t)+3 \psi_{4}(t)+ & \delta_{2}\left[\delta_{2} z(t)+\psi_{2}(t)\right]+2 \delta_{4} z(t)+\delta_{4}\left[2 z(t)-\delta_{4} z(t)-\psi_{4}(t)\right] \\
= & 4 z(t) .
\end{aligned}
$$

(e) $2 z(t)-\delta_{3} z(t)-\psi_{3}(t)-\delta_{2} w(t)-\psi_{2}(t) \neq 0$ and $2 w(t)-\delta_{4} w(t)-\psi_{4}(t)-$ $\delta_{1} w(t)-\psi_{1}(t) \neq 0$.

For each $z, w \in X$ and $t \in[a, b]$, we have

$K_{z w}(t) \sqrt{1+a^{2}} e^{i \tan ^{-1} a} \precsim \alpha \max _{t \in[a, b]} L_{z w}(t)+\beta \max _{t \in[a, b]} M_{z w}(t)+$ $\gamma \max _{t \in[a, b]} N_{z w}(t)$

where,

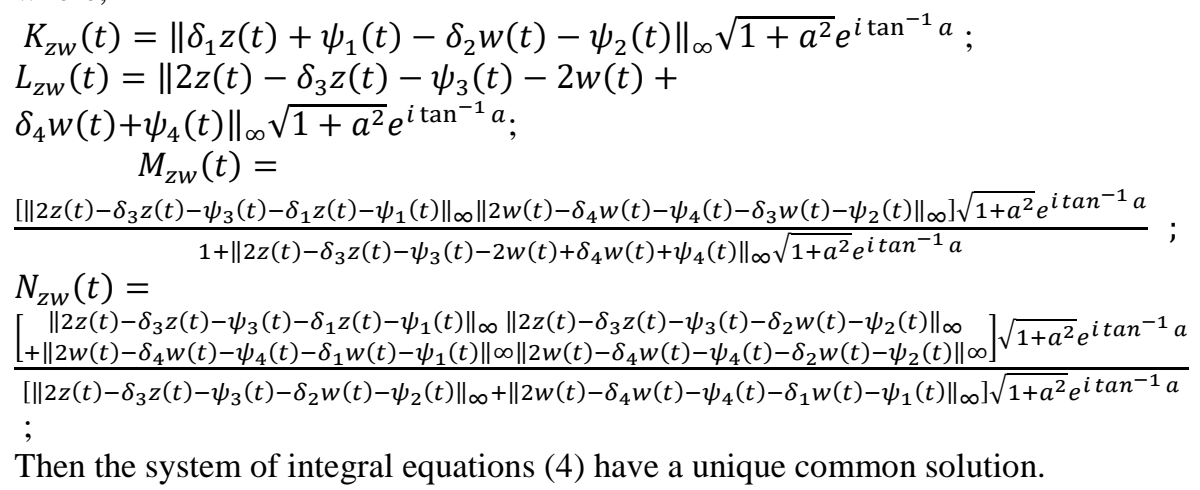

Proof. Define mappings $A, B, S$, and $T: X \rightarrow X$ by

$S z(t)=\delta_{1} z(t)+\psi_{1}(t)=\int_{a}^{b} K_{1}(t, s, z(s)) d s+\psi_{1}(t) ;$
$T z(t)=\delta_{2} z(t)+\psi_{2}(t)=\int_{a}^{b} K_{2}(t, s, z(s)) d s+\psi_{2}(t) ;$
$A z(t)=2 z(t)-\delta_{3} z(t)-\psi_{3}(t)=2 z(t)-\int_{a}^{b} K_{3}(t, s, z(s)) d s+\psi_{3}(t) ;$
$B z(t)=2 z(t)-\delta_{4} z(t)-\psi_{4}(t)=2 z(t)-\int_{a}^{b} K_{4}(t, s, z(s)) d s+\psi_{4}(t) ;$

Let $z, w \in X$, then we get

$d(A z, B w)=$

$\max _{t \in[a, b]}\left\|2 z(t)-\delta_{3} z(t)-\psi_{3}(t)-2 w(t)+\delta_{4} w(t)+\psi_{4}(t)\right\|_{\infty} \sqrt{1+a^{2}} e^{i \tan ^{-1} a}$ $d(A z, S z)=$ $\max _{t \in[a, b]}\left\|2 z(t)-\delta_{3} z(t)-\psi_{3}(t)-\delta_{1} z(t)-\psi_{1}(t)\right\|_{\infty} \sqrt{1+a^{2}} e^{i \tan ^{-1} a}$ $d(A z, T w)=$ $\max _{t \in[a, b]}\left\|2 z(t)-\delta_{3} z(t)-\psi_{3}(t)-\delta_{2} w(t)-\psi_{2}(t)\right\|_{\infty} \sqrt{1+a^{2}} e^{i \tan ^{-1} a}$ 
$d(B w, S z)=$

$\max _{t \in[a, b]}\left\|2 w(t)-\delta_{4} w(t)-\psi_{4}(t)-\delta_{1} w(t)-\psi_{1}(t)\right\|_{\infty} \sqrt{1+a^{2}} e^{i \tan ^{-1} a}$

$d(B w, T w)=$

$\max _{t \in[a, b]}\left\|2 w(t)-\delta_{4} w(t)-\psi_{4}(t)-\delta_{2} w(t)-\psi_{2}(t)\right\|_{\infty} \sqrt{1+a^{2}} e^{i \tan ^{-1} a}$

By the above equation (5) we get

$d(S z, T w) \lesssim$

$\alpha d(A z, B w)+\beta \frac{d(A z, S z) d(B w, T w)}{1+d(A z, B w)}+\gamma \frac{d(A z, S z) d(A z, T w)+d(B w, S z) d(B w, T w)}{d(A z, T w)+d(B w, S z)}$

Now we shall show that $S(X) \subseteq B(X)$. For this

$$
\begin{aligned}
& B\left(S z(t)+\psi_{4}(t)\right)=2\left[S z(t)+\psi_{4}(t)\right]-\delta_{4}\left[S z(t)+\psi_{4}(t)\right]-\psi_{4}(t) \\
& =S z(t)+S z(t)+\psi_{4}(t)-\delta_{4}\left[S z(t)+\psi_{4}(t)\right] \\
& \quad=S z(t)+\delta_{1} z(t)+\psi_{1}(t)+\psi_{4}(t)-\delta_{4}\left[\delta_{1} z(t)+\psi_{1}(t)+\psi_{4}(t)\right]
\end{aligned}
$$

Using the given condition of Theorem (28) we get $B\left(S z(t)+\psi_{4}(t)\right)=S z(t)$ which shows that

$S(X) \subseteq B(X)$. Similarly we show that $T(X) \subseteq A(X)$.

Now, we shall prove that the pair $(S, A)$ and $(T, B)$ are compatible.

Let $\left\{z_{n}\right\}$ be a sequence such that $\lim _{n \rightarrow \infty} S z_{n}(t)=\lim _{n \rightarrow \infty} A z_{n}(t)=z(t)$ for some $z(t) \in X$.

Then we have,

$$
\begin{aligned}
& \left\|S A z_{n}(t)-A S z_{n}(t)\right\|=\left\|S\left(2 z_{n}(t)-\delta_{3} z_{n}(t)-\psi_{3}(t)\right)-A\left(\delta_{1} z_{n}(t)+\psi_{1}(t)\right)\right\| \\
& =\| \delta_{1}\left(2 z_{n}(t)-\delta_{3} z_{n}(t)-\psi_{3}(t)\right)+\psi_{1}(t)-2\left(\delta_{1} z_{n}(t)+\psi_{1}(t)\right)- \\
& \delta_{3}\left(\delta_{1} z_{n}(t)+\psi_{1}(t)\right)-\psi_{3}(t) \| \\
& \quad=\| \delta_{1}\left(2 z(t)-\delta_{3} z(t)-\psi_{3}(t)\right)+\psi_{1}(t)-2\left(\delta_{1} z(t)+\psi_{1}(t)\right)- \\
& \delta_{3}\left(\delta_{1} z(t)+\psi_{1}(t)\right)-\psi_{3}(t) \| \\
& \left\|S A z_{n}(t)-A S z_{n}(t)\right\|=\|-\delta_{1}\left(\delta_{3} z(t)+\psi_{3}(t)\right)-\psi_{1}(t)-\left[\delta _ { 3 } \left(\delta_{1} z(t)+\right.\right. \\
& \left.\left.\psi_{1}(t)\right)+\psi_{3}(t)\right] \| .
\end{aligned}
$$

From condition (c) we get $\left\|S A z_{n}(t)-A S z_{n}(t)\right\|=0 \quad$ whenever $\lim _{n \rightarrow \infty} S z_{n}(t)=\lim _{n \rightarrow \infty} A z_{n}(t)=z(t)$

for some $z(t) \in X$. Hence the pair $(S, A)$ is compatible. Similarly we can show that $(T, B)$ is compatible.

Next we shall prove that the pair $(S, A)$ and $(T, B)$ are weakly compatible. For each $t \in[a, b]$.we get

$$
\begin{aligned}
& \|A S z(t)-S A z(t)\|=\left\|A\left(\delta_{1} z(t)+\psi_{1}(t)\right)-S\left(2 z(t)-\delta_{3} z(t)-\psi_{3}(t)\right)\right\| \\
& \quad=\| 2\left(\delta_{1} z(t)+\psi_{1}(t)\right)-\delta_{3}\left(\delta_{1} z(t)+\psi_{1}(t)\right)-\psi_{3}(t)-\delta_{1}(2 z(t)- \\
& \left.\delta_{3} z(t)-\psi_{3}(t)\right)+\psi_{1}(t) \|
\end{aligned}
$$

If $S z=A z$ for some $z \in X$, then we have $\delta_{1} z(t)+\psi_{1}(t)=2 z(t)-$ $\delta_{3} z(t)-\psi_{3}(t)$ for $t \in[a, b]$.

Therefore from the equation (7), we get

$\|A S z(t)-S A z(t)\|=\| 4 z(t)-2 \delta_{3} z(t)-3 \psi_{3}(t)-\delta_{3}\left(2 z(t)-\delta_{3} z(t)-\right.$ $\left.\psi_{3}(t)\right)-\delta_{1}\left(2 z(t)-\psi_{1}(t)\right)-\psi_{1}(t) \|$ for all $t \in[a, b]$. From condition $(d)$ $\|A S z(t)-S A z(t)\|=0$, that is $A S z(t)=S A z(t)$ for all

$t \in[a, b]$. Therefore $A S z=S A z$ whenever $S z=A z$. 
Hence the pair $(S, A)$ is weakly compatible. Similarly we can show that $(T, B)$ is weakly compatible. Thus all the conditions of Theorem (23) are satisfied. Therefore there exists a unique common fixed point of $A, B, S$ and $T$ in $X$ and consequently there exist a unique common solution of the system of integral equations (4).

\section{Conclusion}

In this article, we extended the study of fixed point theory by using the notions of compatibility and weakly compatibility of self mappings satisfying the new generalized rational type contractive conditions for four self mappings in the complete complex valued $b$-metric spaces. Our results generalized some earlier results exists in the literature. An illustrative example is also given to substantiate our newly proved results. Moreover we demonstrated an application in support of our main result. This idea is expected to bring wider applications of fixed point theorems which will be helpful for researchers to work in the development of fixed point theory.

\section{References}

1. S. Banach, Fund. Math. Monthly 3, 133 (1922). https://doi.org/10.4064/fm-3-1-133-181

2. G. Jungck, Am. Math. Monthly 83, 261 (1976). https://doi.org/10.2307/2318216

3. S. Sessa, Publications DE L'Institut Mathematique 32, 149 (1982).

4. G. Jungck, Inter. J. Math. Math. Sci. 9, 771 (1986). https://doi.org/10.1155/S0161171286000935

5. G. Jungck, Proc. Amer. Math. Sec. 103, 97 (1988). https://doi.org/10.1090/S00029939-1988-0947693-2

6. G. Jungck and B. E. Rhoades, Ind. J. Pure Appl. Math. 29, 227 (1998).

7. A. Azam, B. Fisher, and M. Khan, Numer. Funct. Anal. Optimizat. 32, 243 (2011). https://doi.org/10.1080/01630563.2011.533046

8. I. A. Bakhtin, Funct. Anal., Gos. Ped. Inst. Unianowsk 30, 26 (1989).

9. K. P. Rao, P. R. Swamy, and J. R. Prasad, Bul. Math. Stat. Res. 1 (2013).

10. A. A. Mukheimer, Scientific World J. 2014, 587825 (2014). https://doi.org/10.1155/2014/587825

11. A. K. Dubey, M. Tripathi, and R. Dubey, Int. J. Eng. Math. 2016, ID 7072606 (2016). https://doi.org/10.1155/2016/7072606

12. V. Bairagi, V. H. Badshah, and A. Pariya, Aryabhatta J. Math. Informatics 9, 201 (2017).

13. A. K. Dubey, M. Tripathi, and M. D. Pandey, Asian J. Math. Applicat. 2019, ama0503 (2019).

14. S. Bhatt, S. Chaukiyal, and R. C. Dimri, Int. J. Math. Sci. Appl. 1, 1385 (2011).

15. F. Rouzkard, and M. Indaad, Comput. Math. Applicat. 64, 1866 (2012). https://doi.org/10.1016/j.camwa.2012.02.063

16. S. Dutta and S. Ali, Int. J. Adv. Scientific Technical Res. 6, 467 (2012).

17. S. Chandok, D. Kumar, J. Operators 2013, 813707 (2013). https://doi.org/10.1186/16871812-2013-28

18. W. Situnuvarat, H. K. Nashine, R. P.Agrawal, and P. P. Murthy, J. Math. Anal. 9, 123 (2018). 